\title{
Transposition
}

Musique et Sciences Sociales

1 | 2011

Polyphonie et société

\section{La voix féminine dans les motets français à deux et trois voix du XIII ${ }^{\mathrm{e}}$ siècle}

The female voice in French two and three voice motets in the XIII ${ }^{\text {th }}$ century

\section{Rachel Méegens}

\section{(2) OpenEdition}

\section{Journals}

Édition électronique

URL : http://journals.openedition.org/transposition/110

DOI : 10.4000/transposition. 110

ISSN : 2110-6134

Éditeur

CRAL - Centre de recherche sur les arts et le langage

Référence électronique

Rachel Méegens, "La voix féminine dans les motets français à deux et trois voix du XIII siècle", Transposition [En ligne], 1 | 2011, mis en ligne le 01 février 2011, consulté le 19 avril 2019. URL : http:// journals.openedition.org/transposition/110; DOI : 10.4000/transposition.110

Ce document a été généré automatiquement le 19 avril 2019

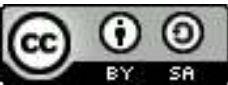

La revue Transposition est mise à disposition selon les termes de la Licence Creative Commons Attribution - Partage dans les Mêmes Conditions 4.0 International. 


\title{
La voix féminine dans les motets français à deux et trois voix du XIII ${ }^{\mathrm{e}}$ siècle
}

The female voice in French two and three voice motets in the XIII ${ }^{\text {th }}$ century

\author{
Rachel Méegens
}

1 La mise en présence simultanée de plusieurs voix que propose la polyphonie suppose une véritable virtuosité compositionnelle et interprétative, mise en œuvre dans le motet profane, genre caractéristique du XIIIe siècle français. Ce dernier prend la forme d'une composition à deux, trois ou quatre voix, les voix supérieures énonçant chacune un texte différent, en français, tandis que la teneur, soit la voix la plus grave sur laquelle se bâtit l'édifice sonore, adopte le latin ou le français. La polyphonie y est prétexte à entrelacer plusieurs discours, et se présente par conséquent comme un lieu de rencontre entre différents locuteurs. Le principe de polytextualité qui régit la composition, induit une conception spécifique du cadre d'énonciation, consistant plutôt, en réalité, en la mise en relation de plusieurs cadres d'énonciation par le biais de l'intertextualité.

2 Le cadre d'énonciation, soit l'ensemble des locuteurs, ou transmetteurs, et de ses destinataires, dans le contexte d'un discours, induit une subjectivité, c'est-à-dire une "capacité du locuteur à se poser comme sujet ${ }^{1}$ ». Cette notion suppose celle de focalisation, présentée par Gérard Genette comme l'adoption d'un " point de vue ${ }^{2}$ " pour la narration. Mieke $\mathrm{Bal}^{3}$, à la suite des travaux de Genette, développe l'idée selon laquelle un discours est focalisé par un personnage, lequel énonce un propos selon son point de vue, et non sur lui, ce qui est particulièrement pertinent pour l'analyse d'une polyphonie de textes telle que le motet.

3 Ce dernier invite donc à poser, en fonction de spécificités induites par la pratique d'une poésie chantée à plusieurs voix, la question de l'énonciation, puisque chaque voix possède un locuteur et un ou plusieurs auditeurs fictifs, déterminés par ce texte lui-même, ainsi que des transmetteurs réels, les chanteurs, leur public étant destinataire. L'énonciation a ainsi lieu sur deux plans différents, l'un imaginaire, l'autre réel, ce qui suppose la 
présence d'une subjectivité poétique réclamant à être assumée vocalement et corporellement par les interprètes.

4 Pertinente pour l'analyse de la monodie, dont est issu le motet, l'étude de l'énonciation se révèle propre à éclairer cette forme polyphonique, dans le but de proposer pour elle une définition du corps énonçant, du Moyen Âge à aujourd'hui, en particulier pour ce qui concerne le motet féminin.

5 Le motet féminin apparait comme l'héritier de la chanson de femme, soit « une chanson amoureuse [...] dans laquelle la perspective de la femme est réalisée soit par un monologue, soit par un dialogue, soit par le récit d'un narrateur ${ }^{4} »$, présente chez les trouvères. Il comporte un ou plusieurs textes dont la locutrice est une femme, le ou les autres textes pouvant éventuellement faire intervenir un locuteur masculin.

6 Ce genre fait intervenir la voix féminine, imaginaire et non réelle puisque les textes à focalisation féminine ne sont pas forcément chantés par une voix de femme. Il pose ainsi la question d'une création artistique de la voix féminine, à une époque où les historiens la décrivent comme peu audible dans la société. La parole féminine est en effet régie par certaines règles : l'accès des femmes à la parole publique est marginal, l'idéal étant, même dans la sphère privée, la «taciturnitas, un comportement vertueux qui impose de parler peu, avec mesure et en seul cas de nécessité5. » Dans ce contexte, le motet apparaît comme une forme de stylisation de la parole amoureuse, à la fois celle que les hommes adressent aux femmes, et celles qu'ils placent dans leur bouche, contribuant ainsi à l'élaboration d'un imaginaire féminin.

7 Parce qu'il pose la question du genre, le motet féminin apparaît comme particulièrement propre au développement d'un riche travail compositionnel sur la focalisation et l'énonciation, inscrit dans un contexte historique. Comprendre comment se construit, d'un point de vue linguistique et littéraire, la voix féminine dans le motet nous semble propre à éclairer la manière dont le Moyen Âge construit imaginairement les femmes, et également les possibilités, pour les interprètes actuels, de revisiter cet imaginaire.

Ainsi, nous proposons une approche du motet en fonction de concepts empruntés non à la musique, mais à la linguistique, afin de mettre en évidence la manière dont s'y construit la voix féminine. Nous l'aborderons en fonction d'un corpus de motets féminins anonymes du XIII siècle, puisé parmi ceux édités par Eglal Doss-Quinby, Joan Tasker Grimbert, Wendy Pfeffer et Elizabeth Aubrey dans leur ouvrage intitulé Songs of the Women Trouvères ${ }^{6}$, soit six motets à deux voix et onze motets à trois voix, dont nous tirerons des exemples précis qui serviront de fondement à nos réflexions.

9 Nous tenterons d'en comprendre la nature afin de déterminer de quelle manière il peut être réinvesti par les interprètes d'aujourd'hui, en fonction de notions de corporéité empruntées aux arts du spectacle et à l'anthropologie théâtrale. Aussi, notre réflexion s'orientera de manière à penser le corps de l'interprète à la fois dans la situation d'énonciation inhérente au motet profane féminin du XIII ${ }^{e}$ siècle, et en fonction d'une situation de transmission bien actuelle, celle du concert. En effet, cette dernière émane d'une société différente de celle de la production des œuvres : la notion de concert est parfaitement étrangère au Moyen Âge. Nous envisagerons donc le repositionnement de ce type de polyphonie médiévale dans un nouveau contexte culturel, rapprochant un genre musical, le motet féminin, et un cadre contextuel, le concert, sans dénominateur commun. Quelles possibilités les interprètes ont-ils de résoudre cette antinomie, en fonction de quelles potentialités présentes dans le texte poético-musical lui-même? 
10 S'il est typiquement une forme d'expression musicale développée par le nord de la France du XIII siècle, le motet trouve indéniablement ses racines dans la monodie des trouvères, et révèle une pensée du corps énonçant élaborée par ce milieu culturel. Définir cette dernière nous amènera à nous pencher sur la notion de personnage, et à travers elle à établir une typologie de la locution dans le motet féminin à partir de laquelle nous étudierons quelques exemples précis à deux et trois voix, de manière à comprendre le fonctionnement de l'énonciation et de la focalisation dans l'intertextualité.

\section{Le motet français : pratique musicale et corps énonçant}

\section{Définition et historique}

11 Prenant pour modèle le motet latin, duquel il hérite la polytextualité, le motet profane connaît son avènement, selon Richard Hoppin, vers 1225 :

Dès ce moment et pendant un demi-siècle, le motet est devenu non seulement la forme prédominante, mais même pratiquement la seule forme de composition polyphonique. On peut donc à juste titre considérer le motet, sacré ou profane, à texte latin ou français, comme la forme représentative de la polyphonie au XIII ${ }^{\mathrm{e}}$ siècle ${ }^{7}$.

12 Dérivant de la clausule, il rencontre son plein développement dans le contexte socioculturel d'une bourgeoisie montante, réunie autour des Puys et autres évènements artistiques et festifs. Il apparaît comme création esthétique liée à l'arrière-plan social, puisque les fêtes et autres évènements collectifs sont autant d'occasions de pratiquer ce type de polyphonie. L'étude des sources comme celle des travaux musicologiques existant sur le motet montre qu'il tient bel et bien une place de choix dans la vie sociale de son époque, encore que les indications sur les occasions lors desquelles des motets étaient interprétés n'abondent pas. La question se pose encore parmi les musicologues, au point que Richard Hoppin reconnaît qu' ' il est difficile d'imaginer à quelle fonction on destinait de telles pièces ${ }^{8} »$. Jean de Grouchy les définit comme un divertissement réservé à un cercle restreint : «ces chants ne doivent pas être présentés à tout le monde, aux gens communs, car ils n'en saisissent pas toutes les subtilités ni ne se délectent à les entendre, mais ils doivent être réservés aux instruits, qui recherchent les subtilités des arts. Ils sont habituellement chantés en tant qu'ornements de leurs fêtes, comme on le fait des chants dits rondeaux, dans les fêtes laïques du peuple ${ }^{9}$.»Christopher Page en déduit que des réunions festives étaient l'occasion privilégiée d'interpréter de telles pièces, " exécutées lorsque les gens letrees - c'est-à-dire les clercs instruits - étaient réunis ensembles ${ }^{10}$ ». Guillaume de Nangis, moine de Saint-Denis et chroniqueur de Philippe III, rapporte que des motets sont interprétés à l'occasion d'évènements importants de la vie d'une communauté :

Les bourgeois de la ville firent une fête grande et solennelle et garnirent la ville de riches draps de diverses couleurs et de tentures et d'étoffes de soie. Les dames et les jeunes filles s'amusaient à chanter diverses chansons et divers motets ${ }^{11}$.

Le motet apparaît donc comme une musique de divertissement profane, ce qui implique l'idée de jeu vocal avec un matériau musical, textuel et rythmique et suggère également une certaine spontanéité, contredite par la subtilité de cette "production savante ${ }^{12}$ ", mise en évidence par Olivier Cullin ${ }^{13}$. 


\section{De l'énonciation à l'incarnation} musicales au cœur de rassemblements sociaux, ce qui induit une corporalisation, c'est-àdire une mise en corps du texte, de la même façon que l'on peut parler de mise en musique, particulière à ce contexte. Ce sont les corps des protagonistes, qui, au milieu de l'une de ces réunions, deviennent des corps autres, transfigurés par le texte, la musique, la polyphonie, qui, à eux seuls, font advenir, collectivement, avec l'espace qu'ils créent entre eux et autour d'eux, une fiction. D'une nature différente de celle du jeu ou du mystère, portée par une manière d'assumer le corps non théâtrale, sans dispositif scénique, cette fiction dépend de l'appropriation d'une, ou en l'occurrence des subjectivités émanant des discours qui constituent les différents textes du motet. Cette corporalisation induit un mode d'écoute de la polyphonie spécifique au Moyen Âge, et qui ne subsiste pas aujourd'hui, où de telles pièces sont interprétées en concert.

Par ailleurs, le corps médiéval est un corps chrétien : même si nous l'abordons ici sous l'angle de la musique profane, le « Moyen Âge [...] s'instaure autour du corps martyrisé et glorifié du Christ ${ }^{14} »$. Ce corps chrétien, donc, s'envisage selon la problématique, très médiévale, de l'incarnation, laquelle affirme l'existence, dans le corps, d'une part immatérielle qui se manifeste par la voix :

Notre verbe devient donc en quelque façon voix matérielle, assumant cette voix pour se manifester aux hommes de façon sensible, comme le Verbe de Dieu s'est fait chair, assumant cette chair pour se manifester lui aussi aux hommes de façon sensible ${ }^{15}$.

Ce que Jean-Marie Fritz traduit en ces termes : au Moyen Âge, « la voix humaine est au plan anthropologique ce que l'Incarnation est au plan théologique : la voix donne chair à notre intériorité, tout comme le Dieu incarné donne voix au verbe ", un «verbe mental propre à chaque individu ${ }^{16}$ ».

17 La voix ainsi pensée est celle de la prière, mais la question de l'incarnation, abordée par la théologie, se pose de toute évidence dans le contexte profane de la polyphonie.

Avec le motet, nous ne sommes pas à proprement parler dans un univers religieux encore qu'il apparaisse comme un lieu de rencontre entre religieux et profane, entre teneur latine et textes français -, mais plutôt dans celui de la fiction profane. Toutefois, la question théologique de la voix est transposable à la parole profane dans la mesure où celle-ci ramène la question de l'incarnation : il ne s'agit plus de donner chair à sa propre intériorité, mais à un personnage.

19 Cette notion de personnage, au fondement d'une analyse de la focalisation et de l'énonciation, y réclame par conséquent d'être définie d'une part en fonction de la polyphonie, et d'autre part selon le contexte médiéval. Ce dernier méconnaît l'individu : « la notion utilisée par le christianisme est celle de personne, mais elle est ambiguë, riche de tensions contradictoires : loin d'exalter d'abord la conscience individuelle, elle tend à abolir le sujet dans la divinité dont il est l'image et dans l'humanité dont il partage le destin ${ }^{17}$.» Dans ces conditions, comment passe-t-on de la notion de personne à celle de personnage ? Rappelons que pour Marcel Mauss, la persona latine ${ }^{18}$ renvoie, à travers son étymologie per sonare au masque à travers lequel résonne la voix de l'acteur dans l'antiquité romaine :

Transposition, 1 | 2011 
Équivalent du grec prosôpon, qui désigne «ce qui est placé devant la vue », persona appartient au champ sémantique de la vision. Il signifie d'abord le masque, puis le rôle joué par un "personnage » : celui qui porte le masque de l'ancêtre pour jouer son rôle ${ }^{19}$. elle n'existe qu'au sein d'un tout formé par l'ensemble des voix. Si l'on définit avec Pierre $\mathrm{Bec}^{20}$ et Michel Zink le XIII ${ }^{\mathrm{e}}$ siècle comme celui du développement d'une «subjectivité littéraire ${ }^{21}$ ", au sens non de la fusion entre l'auteur et le sujet du texte, mais de la construction d'un sujet parlant dont le texte, éventuellement, adopte le point de vue, il semble que la fiction polytextuelle renouvelle la question de l'incarnation.

\section{De la chanson de femme au motet féminin}

\section{La filiation entre chanson et motet à travers la typologie des personnages}

Quant à celle du personnage féminin, elle se profile à travers la notion de personnage telle que la construisent les trouvères, aussi bien dans la monodie que dans la polyphonie :

Le XIII ${ }^{\mathrm{e}}$ siècle témoigne d'une relation nouvelle entre des activités musicales qui jusqu'alors avaient suivi des chemins différents. Dès l'origine, la polyphonie était le domaine réservé des musiciens d'église qui en faisaient usage pour orner le chant liturgique, notamment les tropes et les séquences, et pour mettre en musique des poèmes latins religieux ou moralisateurs. La musique des délassements profanes en langue vernaculaire, d'autre part, se cantonne presque exclusivement dans la monodie. Avec l'apparition du motet profane français au XIII ${ }^{\mathrm{e}}$ siècle, les deux voies se sont rapprochées ${ }^{22}$.

Les points de rencontre en sont par exemple l'œuvre de trouvères comme Adam de la Halle, qui a pratiqué à la fois l'une et l'autre, ou encore le réemploi de chansons ou de fragments de chansons dans le motet. Onques n'amai/Sancte Germane apparaît comme un exemple représentatif d'un tel procédé, puisque la voix de motetus n'est autre qu'un fragment de chanson de femme.

En outre, les voix du motet fonctionnent, considérées indépendamment les unes des autres, à la manière de monodies, dont elles reprennent les topoi et le langage poétique.

Par ailleurs, les types de personnages féminins identifiables dans le répertoire des motets français sont ceux que Pierre Bec a classifiés dans le cadre de la lyrique profane ${ }^{23}$. Ceux qui peuplent les pièces de notre corpus correspondent aux catégories déterminées par lui. Ces œuvres exploitent le thème de la chanson d'ami, «type primitif de la chanson de femme ${ }^{24}$ ", qui « met en scène une jeune fille qui chante sa joie d'avoir un ami qui l'aime, ou, plus fréquemment, se plaint de n'en n'avoir point ou que celui-ci l'ait trahie ${ }^{25}$ ", celui des chansons de départies ou de délaissées, abandonnées par leurs bien-aimés, qui dérive du précédent, ou encore celui des chansons de filles à la fontaine, qui se déroulent au bord de l'eau. Quant à la malmariée, cette "femme malheureuse en ménage et qui se plaint, soit de sa condition en soi, soit de son mari qu'elle méprise pour diverses raisons ${ }^{26}$

Transposition, 1 | 2011 
", nous la rencontrons également à plusieurs reprises, comme le montre le tableau suivant :

Tableau $n^{\circ} 1$ : Typologie des personnages féminins

\begin{tabular}{|c|c|c|c|}
\hline Malmariées & $\begin{array}{l}\text { Délaissées/ } \\
\text { départies }\end{array}$ & Fille à la fontaine & Jeune fille \\
\hline $\begin{array}{l}\text { Je me dois bien } \\
\text { doloseir/Por }\end{array}$ & $\begin{array}{l}\text { Je ne quiers/ } \\
\text { Dieus/Misit }\end{array}$ & $\begin{array}{l}\text { Amours qui vient par } \\
\text { mesage/Toute soule } \\
\text { passerai li bois ramé/ } \\
\text { Notum }\end{array}$ & $\begin{array}{l}\text { Qu'ai je forfait ne mespris/ } \\
\text { Bons amis/In seculum }\end{array}$ \\
\hline $\begin{array}{l}\text { Je sui jonete et jolie/ } \\
\text { Hé Dieux !/Veritatem }\end{array}$ & $\begin{array}{l}\text { Quant se depart/ } \\
\text { Hé! cuer joli/In } \\
\text { seculum }\end{array}$ & & $\begin{array}{l}\text { Cil bruns ne me meine mie/ } \\
\text { In seculum }\end{array}$ \\
\hline \multirow[t]{9}{*}{$\begin{array}{lr}\text { Amis, } & \text { vostre } \\
\text { demoree/Pro patribus }\end{array}$} & $\begin{array}{l}\text { Onques n'amai/ } \\
\text { Sancte Germane }\end{array}$ & & Je les ai tant quises/Vitam \\
\hline & & & $\begin{array}{l}\text { J'ai fait ami a mon chois/ } \\
\text { Gaudete }\end{array}$ \\
\hline & & & $\begin{array}{l}\text { À vos vieg, chevalier sire/Et } \\
\text { florebit }\end{array}$ \\
\hline & & & $\begin{array}{l}\text { Biauz douz amis, or ne vous } \\
\text { anuit mie/Domine }\end{array}$ \\
\hline & & & $\begin{array}{l}\text { Diex! De chanter } \\
\text { maintenant/Chant d'oisiaus } \\
\text { et fuelle et flors/In seculum }\end{array}$ \\
\hline & & & $\begin{array}{l}\text { Qu'ai-je forfait ne mespris/ } \\
\text { Bons amis, je vos rendrai/In } \\
\text { seculum }\end{array}$ \\
\hline & & & $\begin{array}{l}\text { Mout me fut grief/Robin } \\
\text { m'aime/Portare }\end{array}$ \\
\hline & & & $\begin{array}{l}\text { S'on me regarde/ Prenes i } \\
\text { garde/Hé mi enfant }\end{array}$ \\
\hline & & & $\begin{array}{l}\text { Dame que je n'os noumer/ } \\
\text { Amis, dont est engenree/ } \\
\text { Lonc tans a que ne vis ma } \\
\text { mie }\end{array}$ \\
\hline
\end{tabular}

La survivance au sein du motet des types de personnages développés par la chanson constitue donc un lien entre polyphonie et monodie, qui permet d'y envisager l'étude de la focalisation et de la subjectivité du personnage parlant à partir de cette dernière. 


\section{La notion de personnage chez les trouvères} d'une action, en interaction avec d'autres personnages. Au contraire, ni la chanson ni le motet, en raison de la brièveté de leurs formes, ne sont à même de développer l'individualité de leurs protagonistes, ni de leur accorder de véritable épaisseur psychologique. Ils demeurent plutôt des types, à l'inverse des héros romanesques ou dramatiques. Contrairement à ces derniers, le personnage de la lyrique se définit exclusivement par l'énonciation et la focalisation, ne bénéficiant pas de descriptions comme un personnage de roman, non plus que le chanteur médiéval, comme nous le verrons par la suite, ne dispose des ressources de l'acteur de théâtre que sont les gestes ou le jeu de scène. Ainsi, la malmariée, la jeune fille, la délaissée, etc. de la chanson tiendront toujours, plus ou moins, le même discours, qui se perpétue dans les voix du motet.

\section{Polyphonie et subjectivité}

\section{Polyphonie, personnage, fiction}

31 La polyphonie telle qu'elle se conçoit dans le motet implique une accumulation de discours simultanés.

Dans la tradition latine, le rapport entre les voix est fortement perceptible. Traitant d'un même sujet, elles sont complémentaires ; citons par exemple le motet Candida Virginitas/ Flos filius eius, qui développe le thème de la prophétie de l'arbre de Jessé. Cette conception de la complémentarité des textes induit une pensée "spectrale » du corps, au sens d'un spectre sonore, tant les voix sont mises en résonance les unes par rapport aux autres par

Transposition, 1 | 2011 
le sens des mots. Si nous entendons deux ou trois voix, nous voyons autant de corps spectraux, issus de la diffraction d'un corps originel, la voix, au sens propre comme au figuré, de teneur.

Ces spécificités sont d'autant plus évidentes que l'énonciation est historique, et non discursive : elle consiste en l'exposé de faits bibliques. Mais cette problématique texte/ voix/corps est transposable au motet profane, qui, au contraire, relève du discours. La complémentarité des textes y est également de mise. Toutefois, ce n'est plus le sens, la thématique, qui créent le phénomène spectral, mais la notion de personnage, et plus précisément la notion de type, laquelle fait le lien entre les textes, entre les voix, entre les corps, comme nous le constaterons plus loin.

La multiplicité des textes va de pair avec la multiplicité des locuteurs, fictifs, et par conséquent celle des voix énonçantes, celles des interprètes.

Toute musique vocale prenant la forme d'un discours imaginaire (air d'opéra, mélodie...) suppose que l'interprète assume le personnage fictif dont il s'approprie le discours. Théâtral, l'opéra est fondé sur un principe de simultanéité entre le corps et la voix, dans un but illusionniste. En revanche, les genres vocaux non théâtralisés s'inscrivent dans une forme de concert héritée du XIXe siècle : le récital. Quels sont les enjeux soulevés par l'introduction au cœur du dispositif d'un tel rituel socioculturel, d'un genre polyphonique médiéval comme le motet, qui porte intrinsèquement la question de la théâtralité, à travers celle de l'incarnation?

Pierre Bec a défini la lyrique comme le genre le moins théâtral qu'ait produit le Moyen Âge, car elle apparaît comme une «négation fondamentale de la spatialité et de l'action ${ }^{29}$ ». Ce constat pourrait apparaître d'autant plus vrai dans le cas du motet, étant donné le mode de parole polyphonique qui le caractérise.

Or il adopte un modèle discursif qui l'apparente au lied, à la mélodie, et qui était déjà, d'ailleurs, celui de la monodie, tout en proposant la complexification de ce modèle par la présence de plusieurs textes simultanément, laquelle autorise la pluridimensionnalité de la fiction, voire la multiplicité des fictions, que crée l'ensemble des voix : chacune d'elle apparait comme une part d'un tout fictionnel en même temps que de l'édifice sonore.

Ainsi, la polyphonie propose une autre forme d'incarnation du personnage construite dans la vocalité, et non sur un corps imitatif : la fiction est dite et non jouée, et demeure dans une dimension imaginaire créée par la rencontre des voix, des textes, réalisée par la polyphonie.

\section{Personnage et interprète}

Les chanteurs pratiquant ce type de polyphonie ne prétendent pas incarner les personnages fictifs à la manière d'acteurs, ou comme ce sera le cas dans l'opéra. Nous avons pourtant défini un statut particulier du personnage dans le cadre du motet ; reste à déterminer de quelle manière le chanteur l'assume.

Un chanteur, quel que soit le style de la pièce qu'il aborde, opéra, lied, comédie musicale, ou, comme ici, motet médiéval, construit son interprétation selon un ou des référents, plus ou moins conscients, à une réalité hors-scène. Lorsqu'un interprète de notre siècle se trouve face à un motet féminin $\mathrm{du} \mathrm{XIII}^{\mathrm{e}}$ siècle, il est amené, pour nourrir son interprétation, à adopter des références hors-scène, imaginaires, historiques, littéraires, etc. 
41 La chanson monodique propose deux référents différents : le personnage de la chanson, et le trouvère lui-même. Bien que les éléments autobiographiques que comporte volontiers ce type de texte tendent à confondre personnage et trouvère, à les fondre dans une même figure imaginaire, sous l'effet de l'éloignement temporel, il a été largement montré, notamment dans les travaux de Michel Zink, que la valeur autobiographique de la lyrique médiévale est nulle :

Certes, la chanson courtoise se donne pour une confidence de l'amour, mais c'est une confidence « illusoire ». Le poète se contente de proclamer qu'il aime, et il n'y a bien évidemment aucun sens à s'interroger sur la vérité de cette proposition. Il y en a un, en revanche, à définir le rôle joué dans le système poétique par ces protestations de sincérité amoureuse, qui créent l'illusion de la confidence ${ }^{30}$.

Ces chansons proposent à l'interprète un triple référent : le poète, le musicien médiéval, le personnage fictif, posant à l'interprète la question de l'investissement d'une subjectivité autre.

Anonyme, l'auteur du texte ne constitue qu'un référent très vague, qui s'efface devant le ou les sujets parlants, car nombreux sont les motets où, à la faveur de la pluralité des voix, se croisent plusieurs personnages, féminins ou non.

Quant aux chanteurs ayant interprété des motets au Moyen Âge, évoqués dans des textes comme celui de Guillaume de Nangis ${ }^{31}$, ils demeurent, pour les interprètes de notre siècle, un modèle inaccessible en raison d'un certain vide documentaire. Leur interprétation, méconnue étant donné l'imprécision des sources quant à la manière de chanter et de jouer, est, par là même, à l'origine de la construction d'un univers imaginaire et artistique protéiforme, à travers une quête de vraisemblance et d'exactitude historique qui est aujourd'hui la caractéristique essentielle de l'interprétation des ensembles spécialisés.

Le motet, en tant que forme polyphonique, repose donc la question de la monodie : à qui renvoie le «je» du texte? Pierre Bec formule en ces termes cette interrogation fondamentale : « lyrisme = subjectivité ? Oui, si l'on veut dire par là que le sujet objectif de l'effusion est un “"je"' qui se chante et autour duquel toute la pièce s'organise ; non si l'on entend par subjectivité une adéquation spontanée et directe (à la romantique) de l'effusion lyrique aux pulsions effectives du sujet chantant ${ }^{32}$.» Pour Michel Zink, il s'agit d'un « je » fictif, qui joue le jeu du réel à travers "l'illusion de la confidence ", sans relever réellement de l'autobiographie : les tourments amoureux décrits renvoient à une expérience assez commune pour que chacun ait loisir de s'y reconnaître. Emmanuèle Baumgartner voit quant à elle dans cet «écart entre le poète et le poème ${ }^{33}$ »la potentialité $d u$ « je » lyrique à être réinvesti, en d'autres lieux et d'autres temps, par un « je » différent, celui de l'interprète ${ }^{34}$, à qui le texte propose de jouer, à son tour, ce jeu de la confidence, en s'identifiant au personnage.

Dans un contexte polyphonique, il ne s'agit plus pour les interprètes d'identifier un, mais plusieurs «je », et de déterminer quelle est la relation entre eux. Les récents travaux

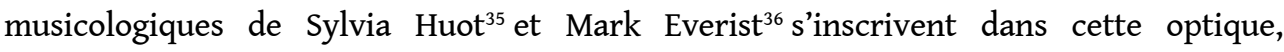
puisqu'ils se donnent pour objectif de mettre en évidence les relations intertextuelles au sein du motet afin d'en éclairer le sens. Plusieurs « je », plusieurs subjectivités, supposent la multiplication des points de vue.

47 En tant que forme savante, abstraite, non dramatique et non mise en scène, le motet ne prétend pas créer l'illusion. Le contenu des textes est typiquement pseudo-narratif, dans la mesure où il donne l'impression de raconter une histoire (celle d'une femme délaissée, 
celle d'une jeune fille et de son ami...) mais se trouve être en réalité plutôt une description, par le personnage principal, de ses états d'âme.

Pourtant, l'appropriation de la subjectivité d'un personnage fictif est contrariée par la présence d'autres voix, d'autres corps sonores, vocaux ou instrumentaux, éventuellement d'autres «je ", lesquels désolidarisent personnage et chanteur. Cette absence d'illusion crée un type d'incarnation propre au motet. Il s'y invente un jeu focal, par la multiplicité des personnages et des subjectivités qui s'y croisent, s'y répondent, y dialoguent. Mais cette question de l'illusion pose surtout celle du degré d'identification entre la voix et le corps, par laquelle l'interprète est amené à explorer les frontières séparant le chanteur de l'acteur.

Aux interprètes, donc, d'inventer un corps pour le motet, une incarnation qui ne se ferait pas dans l'illusion mais à travers un jeu sur différents degrés de solidarité voix-corps. En concert, les chanteurs se trouvent sur scène, face à un public qui les voit en tant que tels, et non en tant qu'acteurs : le personnage, à travers son discours, est assumé par la voix, et non le corps. Il s'agit d'une forte désolidarisation entre voix et corps, puisque seule la première assume le personnage, tandis que le second demeure celui d'un chanteur en concert.

Penser au musicien du Moyen Âge comme à une référence hors-scène autorise les interprètes actuels à dramatiser la forme, à créer autour d'elle mises en espace, mises en scène, gestualisation, "gesticulation ${ }^{37}$ " pour employer le terme d'Eugenio Barba. La forme est alors incluse dans une recréation imaginaire du Moyen Âge, et non seulement de la substance du texte poétique.

51 Pour ce qui concerne le texte, l'écriture polyphonique du motet amplifie les questions posées par la monodie: la présence de plusieurs voix leur apporte une nouvelle dimension, dans la mesure où la pluralité des textes va de pair avec la pluralité des focalisations, et la pluralité de l'énonciation.

\section{Le motet à deux voix}

\section{La notion de subjectivité dans le motet à deux voix}

Ces réflexions nous amènent à tenter de définir la subjectivité telle qu'elle existe dans le motet à deux voix, afin d'examiner ce que cette définition induit dans le cadre d'une représentation.

Dans ces motets, qui comportent une teneur en valeurs longues et une voix de motetus, ou duplum, en français, la focalisation fonctionne sensiblement comme celle de la chanson. En effet, la teneur, le plus souvent en latin, parfois en français, ne comporte pas de texte ; seul l'incipit apparaît dans le manuscrit. Aussi existe-t-il deux choix possibles d'interprétation: soit le ou les chanteurs qui interprètent la teneur en prononcent le texte; soit ils la chantent sur une syllabe neutre, approchant ainsi le son d'un instrument. L'un et l'autre choix sont populaires parmi les interprètes. Chantée sur le mode instrumental, la teneur est en quelque sorte entendue comme une voix qui ne dit rien, qui n'énonce pas. Même chantée cum littera, la teneur ne dit pas "je ». En effet, la nature religieuse de son texte en latin implique une énonciation historique et non discursive, dont est absente toute subjectivité, ce qui l'exclut du jeu de la focalisation. 

médiévale, d'un spectacle intitulé Rose tres bele, conçu à la manière d'un concert mis en scène et en images, dont le fil conducteur était la musique des dames trouvères, monodique et polyphonique. Faisait notamment partie de ce programme le motet Onquesn'amai/Sancte germane évoqué plus haut. Le choix musical fut de ne pas faire entendre la teneur dès le début de la pièce, mais seulement à partir de la deuxième strophe, sine littera. Son entrée s'accompagnait, visuellement, scéniquement, d'un rapprochement entre les deux corps des chanteuses, la solidarité des corps répondant à la solidarité des voix, l'une énonçante et l'autre non. La proximité des silhouettes facilitait leur assimilation, par opposition aux autres musiciens éparpillés sur la scène, et tendait à montrer un corps dédoublé plutôt que deux corps. sons mais ne dit rien, tel l'«instrument corporel sonore ${ }^{39}$ " défini par Ricardo Jacobsohn. Les deux corps sont solidarisés dans le propos de celui qui parle, faisant du « je » fictif une entité double, et, justement, polyphonique.

choix différent de mise en espace pourrait faire entendre la teneur comme un élément étranger au discours tout en étant simultané, un peu comme le serait l'accompagnement 
instrumental d'une monodie. Ainsi, la polyphonie peut se rapprocher de la monodie accompagnée, notamment lorsque la teneur est jouée par un instrument.

61 Au contraire, une voix instrumentale, c'est-à-dire ne parlant pas, sine littera, s'en éloigne, proposant au spectateur la vision d'un corps sonore, mais non parlant, dont la fonction semblera être de mettre en valeur le corps parlant proprement dit, le motetus. Il porte le texte de ce dernier, et à juste titre, puisque dans la composition du motet, la teneur a pour fonction de porter l'édifice sonore, dont elle constitue la fondation.

Enfin, dans le cas où la teneur est chantée cum littera, la divergence avec le motetus s'affirme, puisque l'on entend deux voix en même temps, et que l'on voit deux corps tenir des propos différents. La mise en évidence de la solidarité est moindre, l'unicité que l'on imagine dans les autres cas n'a plus lieu d'être, où du moins s'entend autrement ; il s'agit toujours d'une composition unitaire.

Les interprètes sont donc amenés à imaginer un corps pour la polyphonie : le choix d'une teneur vocale ou instrumentale, d'une teneur « parlante » ou non, n'est pas uniquement un paramètre sonore, mais détermine cette corporéité.

\section{Le motet à trois voix}

Le motet à trois voix, ou motet double, adjoint une voix de triplum au motetus et à la teneur.

Cette dernière présente les mêmes caractéristiques et les mêmes potentialités que dans un motet à deux voix, tandis que le couple motetus-triplum permet un nouveau jeu sur l'énonciation et la focalisation par le biais de la polytextualité, laquelle ouvre la perspective de la complémentarité des textes suggérée par les travaux de Mark Everist ${ }^{40}$ et de Sylvia Huot: « le motet, bâti sur des textes indépendants exécutés simultanément, offre une opportunité de jeu intertextuel sans égal dans les autres formes littéraires ${ }^{41}$. »

En l'occurrence, le motet féminin développe de manière pluridimensionnelle la ou les situations-thèmes typiques de la monodie, à savoir celle de la malmariée trompant ou projetant de tromper son mari, celle de la jeune fille délaissée par son ami, ou encore celle de jeunes gens amoureux... donnant la parole à l'un, ou l'autre, ou les deux, des principaux protagonistes. Le motet double se décline donc en deux types: les motets à locution féminine, et ceux à locution mixte. Parmi les premiers, les uns comportent une seule locutrice, malgré la présence de deux voix, et les autres deux. Les seconds, les plus nombreux, naissent de la rencontre entre voix masculine et voix féminine.

Tableau $n^{\circ} 2$ : Répartition des types de locution

\begin{tabular}{|l|l|l|}
\hline Motets à locuteur unique & $\begin{array}{l}\text { Motets à locution } \\
\text { féminine }\end{array}$ & Motets à locution mixte \\
\hline $\begin{array}{l}\text { S'on me regarde/ Prenes } \\
\text { i garde/Hé mi enfant }\end{array}$ & $\begin{array}{l}\text { Je sui jonete et jolie/Hé } \\
\text { Dieus !/Veritatem }\end{array}$ & $\begin{array}{l}\text { Diex! De chanter maintenant/Chant } \\
\text { d'oisiaus et fuelle et flors/In seculum }\end{array}$ \\
\hline Je ne quier/Dieus/Misit & $\begin{array}{l}\text { Qu'ai-je forfait ne mespris/Bons amis, je } \\
\text { vos rendrai/In seculum }\end{array}$ \\
\hline & Mout me fut grief/Robin m'aime/Portare \\
\hline
\end{tabular}




\begin{tabular}{|l|l|l|}
\hline & & $\begin{array}{l}\text { Je me dois bien doloseir/Por coi m'aveis } \\
\text { vos doneit/Docebit }\end{array}$ \\
\hline & $\begin{array}{l}\text { Nus ne mi pourroit conforter/ Nonne sui/ } \\
\text { Aptatur }\end{array}$ \\
\hline & $\begin{array}{l}\text { Dame que je n'os noumer/Amis, dont est } \\
\text { engenree/Lonc tans a que ne vis ma mie }\end{array}$ \\
\hline & Quant se depart/Hé! cuer joli/In seculum \\
\hline & & $\begin{array}{l}\text { Amours qui vient par mesage/Toute soule } \\
\text { passerai li bois ramé/Notum }\end{array}$ \\
\hline
\end{tabular}

\section{Les motets à locution mixte}

L'écriture caractéristique à trois voix autorise la mise en présence des deux genres, masculin et féminin, au sein de la même pièce, ce qui marque une évolution par rapport à la chanson monodique, dans laquelle seule la voix de l'interprète portait le ou les cadres d'énonciation et assumait les éventuels changements de focalisation.

La monodie ne développe qu'un seul cadre d'énonciation, qu'une seule focalisation à la fois. Le locuteur assume, en théorie, toutes les voix fictives au moyen de sa propre voix réelle, c'est-à-dire qu'il incarne tous les personnages présents dans la pièce. Pour ce qui est des changements de focalisation dans la monodie, la plupart des chansons de femmes sont focalisées sur la seule héroïne, qui garde la parole du début à la fin; dans certaines autres, un autre locuteur intervient, son discours alternant avec celui de l'héroïne. Ces changements de point de vue concernent fréquemment, pour ce qui est de la lyrique, un narrateur et le personnage féminin, et excluent les personnages masculins, ami, mari ou jaloux.

$69 \mathrm{Au}$ contraire, les polyphonistes jouent sur la capacité du motet triple à mettre en présence deux locuteurs, le motetus et le triplum, dédoublant ainsi la focalisation. Cette simultanéité est à même de créer une fiction pseudo-narrative en raccourci, en confrontant les discours de deux personnages.

70 Pour détailler notre propos, nous choisirons deux exemples de motets à trois voix : Je me dois bien doloseir/Por coi m'aveis vos doneit/Docebit d'une part, et Mout me fu grief/Robin m'aime/Portare d'autre part ${ }^{42}$.

71 Dans le premier, le texte du motetus est celui d'une malmariée, tandis que celui du triplum est celui d'un homme se plaignant du fait que sa bien-aimée soit mariée à un autre. Le rapprochement de ces deux textes au cœur de la même œuvre suggère qu'à la voix de l'une répond celle de l'autre. La complémentarité des deux voix, qui participent d'une structure polyphonique complexe, d'un échafaudage sonore, laisse entendre une intrication équivalente des textes: la voix masculine est entendue comme celle de l'ami que la femme évoque dans son discours, tandis que la voix féminine tient la place de celle de «la belle» évoquée par l'ami malheureux. L'individualité peu développée des personnages, demeurés à l'état de types, les dote d'une certaine universalité : il peut s'agir de n'importe quelle femme et de n'importe quel ami. Ainsi, la pièce renvoie 
éventuellement à d'autres œuvres littéraires ou poétiques du Moyen Âge, chansons ou romans, selon un principe de création typique de cette époque, selon lequel le créateur revisite en permanence des créations préexistantes: «le texte médiéval n'a pas pour point de départ la personnalité de son auteur, mais un archi-texte réel ou fictif ${ }^{43}$.»

La voix féminine fonctionne ici par rapport à la voix masculine, sur une teneur neutre. Cette construction fictionnelle pose la question de l'intelligibilité, compromise par la prononciation des deux textes en même temps. De plus, cette simultanéité interdit tout dramatisme ; elle se prête particulièrement au principe de la fiction pseudo-narrative, et aussi à la nature du "personnage de motet " tel que nous l'avons défini plus haut. Le rapprochement de ces deux textes suggère une véritable saynète, mettant en présence la malmariée et son ami. Cette idée de saynète ramène de manière concrète la question de la théâtralité du motet, théâtralité extérieure à la fiction, qui réside dans la vision des corps virtuoses mais non imitatifs. Même s'il n'y a pas représentation au sens théâtral que l'on prête à ce terme, la musique, le chant, existent dans la représentation, dans la " performance ${ }^{44}$ » dont parle Paul Zumthor, « instance de symbolisation; d'intégration de notre relativité corporelle dans l'harmonie cosmique signifiée par la voix ; d'intégration de la multiplicité des changes sémantiques dans l'unicité d'une présence ${ }^{45}$ ». Les fêtes données par les bourgeois de Paris sont des situations de représentation, le concert tel que nous le connaissons aujourd'hui en est une autre. La clé en est le corps de l'interprète, dans la mesure où, en l'absence d'intention illusionniste, se crée une corporalisation autre que celle du théâtre.

La polytextualité autorise l'inclusion de références à d'autres œuvres, organisant "plusieurs motifs déjà connus ${ }^{46}$ ", selon le "principe de composition par "collage" 47 ", mis en évidence par Mikio Katayama.

Par exemple, le motet à locution mixte Mout me fu grief/Robin m'aime/Portare a la particularité de faire intervenir un personnage théâtral, puisque la voix de motetus est un fragment chanté par Marion dans le Jeu de Robin et Marion. Le personnage de théatre fait irruption dans le motet, mais désolidarisé de ce qui constitue son identité dans le théâtre: sa place dans une action, l'interaction avec les autres personnages, l'épaisseur psychologique acquise au cours d'une action dramatique d'ampleur... Faut-il donc tenir compte de ce statut de personnage de jeu dans l'analyse de ce motet?

La référence à Robin présente dans le texte, la mélodie bien connue d'Adam de la Halle agissent comme des références qui éveillent, dans l'oreille de l'auditeur averti, l'écho du jeu : il ne s'agit pas de n'importe quelle jeune fille, le Jeu de Robin et Marion étant une référence connue de la musique médiévale. En effet, Louis Monmerqué décrit cette œuvre comme fort bien diffusée au XIII siècle $^{48}$. Par ailleurs, les nombreuses éditions dont elle a fait l'objet depuis le $\mathrm{XIX}^{\mathrm{e}}$ siècle, ainsi que les diverses publications discographiques plus récentes, sont autant d'indicateurs de sa popularité aux $\mathrm{XX}^{\mathrm{e}}$ et $\mathrm{XXI}^{\mathrm{e}}$ siècles également. Aussi pouvons-nous reprendre à notre compte les réflexions de Mikio Katayama, lequel affirme que l'insertion dans le motet de citations d'autres œuvres permet d'éclairer le sens de la pièce d'un jour nouveau ${ }^{49}$. Ici, la voix de l'ami, qui exprime dans un registre populaire sa tendresse, son " amiete », peut être entendue comme celle de Robin, faisant de la pièce une sorte de glose sur le Jeu de Robin et Marion, ce dernier se trouvant ici en position d'archi-texte. 


\section{Le motet à locution féminine}

76 Un autre choix de focalisation consiste à faire d'une femme l'unique locutrice de la pièce, malgré la duplicité des voix supérieures, la teneur demeurant une « voix qui ne dit rien ». C'est par exemple le cas dans le motet S'on me regarde/Prenés i garde/Hé mi enfant ${ }^{50}$.

Le discours tenu dans ce motet est celui de la chanson de jeune fille, dans un contexte de pastourelle. Partagé entre motetus et triplum, il crée une fiction unitaire autour du personnage féminin. Deux voix, deux corps sonores se font support d'un seul personnage. Le principe de polytextualité est conservé, mais les deux voix supérieures sont dotées de deux textes proches, dont l'un semble la variation de l'autre. En effet, les mots, phrases ou fragments de phrases communs aux deux voix créent un effet de dédoublement. "S'on me regarde », « dites le moi », " prenes i garde », « trop sui gaillarde », « bien l'aperchoi », « tel ci voi », « qui est je croi », « pour nient m'esgarde, bien pert sa garde », « feu d'enfer l'arde », «j'arai rechoi »... : l'effet d'écho créé par ce contrepoint de mots aux sonorités proches joue sur une impression d'unité/duplicité.

Ces expressions répétées, passant d'une voix à l'autre comme les fils d'une tapisserie, mettent en évidence la construction de la pièce autour de l'unité d'un discours, et donc autour de l'unicité d'une hérö̈ne principale. Les autres personnages caractéristiques de l'univers courtois, l'ami et le jaloux, ne sont qu'évoqués à travers sa voix, à travers sa parole, et n'interviennent qu'à travers un jeu de regards avec l'héroïne. Nous pouvons les définir, avec Émile Benveniste, comme des "non-personnes", des "référence[s] zéro hors de la relation $\mathrm{je} / \mathrm{tu}^{51} »$ qui constitue le cadre d'énonciation. Dans ce texte très visuel, la jeune fille se pose à la fois comme objet de regards désigné par les pronoms « moi » ou «me», et comme sujet parlant, pensant, agissant («je croi », «j'arai»). Son discours construit son propre récepteur fictif, un «vous » présent à travers les formes verbales «prenes » et «dites». Ce modèle de cadre d'énonciation dérive de celui qu'installe la monodie, en ce que la pièce adopte un point de vue unique, sans changements de focalisation. Il propose typiquement un corps diffracté : les deux voix réelles, les deux corps chantants ne renvoient qu'à un seul « je » fictif, et ne construisent pas seulement le tout sonore de la pièce mais également le tout subjectif du personnage.

Enfin, le motet à locution féminine met en présence deux textes complémentaires, féminins l'un et l'autre, exprimant des idées ou des sentiments semblables, ou dépeignant des situations identiques, mais dans des discours suffisamment distincts pour que l'auditeur y entende deux personnages appartenant au même type. Le rapprochement entre les deux voix, triplum et motetus, se fait à la fois à travers le sens du discours, dans lequel les deux voix expriment la même idée, ou des idées très proches mais complémentaires, et par le biais des mots employés.

Dans le motet double Je sui jonete et joliete/Hé Diex!/Veritatem, triplum et motetus sont des chants de malmariée ${ }^{2}$.

81 La malmariée du premier prétend aimer son ami malgré la jalousie de son mari, tandis que celle du second se plaint de son mari et de tous ses défauts : elle souhaite avoir un ami pour l'en consoler. À elles deux, ces voix résument la définition de la malmariée proposée par Pierre Bec : «la malmariée médiévale [...] possède déjà un ami, avec lequel elle trompe joyeusement son mari, ou bien elle ne l'a pas encore mais le souhaite au plus vite, en le parant par anticipation de toutes les qualités que n'a pas son époux ${ }^{53}$. » 

elles sont toutes deux malmariées : l'une a un ami, l'autre pas. Cette divergence tend à en faire deux personnages distincts, même si leur langage les rapproche, au moyen du système d'échos déjà rencontré, et courant dans la polyphonie médiévale : «mon mari ", «a mon/son gré ", «j'amerai » sont les mots qui traversent la pièce à l'une et l'autre voix. La teneur elle-même fait écho au propos du triplum, par un rapprochement des sonorités de « vérité » et « veritatem ».

L'ambigüité de ce motet réside donc dans un questionnement sur l'identité de la ou des locutrices : y a-t-il un personnage ou deux?

Il peut être pensé comme l'accumulation de deux pseudo-narrations, ou bien comme une seule et unique narration. On peut également envisager une particularité stylistique, autorisée justement par la polyphonie, un parallélisme selon lequel l'héroöne pourrait à la fois tenir l'un et l'autre propos. Si le rapprochement se fait à travers les termes employés et les sonorités des paroles, l'hétérogénéité des situations, des "scènes", empêche d'identifier à coup sûr motetus et triplum. On peut également comprendre le rapprochement de ces deux voix comme temporel: les deux situations concerneraient alors un même personnage à deux moments différents, celui où elle souhaite avoir un ami, et celui où elle l'a réellement.

Si l'on admet que ces deux voix discursives appartiennent possiblement au même personnage, ou à deux personnages n'étant que des variations d'un type unique, les chanteurs auxquels sont confiés ces discours n'apparaissent pas comme deux individualités, comme deux corps en scène, mais plutôt comme un unique corps dédoublé.

\section{Conclusion}

Interroger la polyphonie médiévale à travers les notions d'énonciation et de focalisation permet donc de prendre en compte les dimensions textuelles et intertextuelles et de révéler une pensée du corps parlant, éclairant, pour le Moyen Âge, la notion de subjectivité. Cette dernière s'y définit par l'absence de fusion entre l'auteur du texte et le sujet parlant qu'il construit. Du sujet parlant dans la dimension imaginaire du texte à la voix de l'interprète qui fait advenir le personnage, c'est la rencontre avec d'autres sujets, d'autres corps sonores, parlants ou non (teneur sine littera) qui fait sens.

Superposition de discours chantés, le motet féminin se conçoit au XIII ${ }^{e}$ siècle comme une polyphonie de textes, de sons, de corps, de voix. Héritière de la monodie, cette forme de polyphonie reprend et développe le modèle d'énonciation que cette dernière proposait: plusieurs textes supposent plusieurs sujets parlants, et donc plusieurs corps énonçants. Cette pluralité pourrait engendrer un éclatement de la forme et du sens, mais elle est au contraire prétexte à ce que les textes superposés bâtissent une unité fictionnelle, notamment autour du personnage. Notre réflexion fait apparaître cette notion de personnage comme essentielle, car l'idée de personnage-type rend possible un jeu de références intertextuelles, mais aussi archi-textuelles, pour reprendre le mot de Michel Stanesco ${ }^{54}$. Les discours font sens individuellement, mais s'éclairent réciproquement et s'enrichissent d'un nouveau sens dans l'interaction, par le jeu de la multiplicité des points de vue. 
88 Entre diffraction et complémentarité, le motet est à même de revisiter la subjectivité construite par la monodie en lui offrant une pluridimensionnalité permise par le nombre de voix, aussi bien qu'à donner simultanément la parole à deux personnages différents, illustrant une manière toute médiévale de concevoir la fiction poético-musicale. La question de l'incarnation, ou mieux, de la corporalisation, soit la mise en corps du texte, rejoint une problématique contemporaine, déjà traitée par l'anthropologie théâtrale, qui réfléchit à la manière dont l'acteur accueille dans son corps le personnage de théâtre. En effet, les interprètes se trouvent aujourd'hui face au défi de confronter le corps parlant tel que l'a défini le Moyen Âge à la situation du concert, issue d'une tradition musicale qui a, elle, conçu un corps chantant: celui du récitaliste. Susan Hellauer, chanteuse de l'ensemble Anonymous 4, résume ainsi les propositions faites par les ensembles spécialisés :

Il y a plusieurs solutions possibles. Pour donner un exemple extrême, des ensembles présentent la musique médiévale dans la tradition du récital de mélodies du XIX siècle. Ils portent un habit de soirée. Ils commencent leurs concerts par saluer sous les applaudissements de leur public. Ils chantent un certain nombre de pièces, recevant des applaudissements après chaque œuvre ou "suite». Leurs concerts comprennent un entracte, et peuvent être suivis d'un ou plusieurs rappels. Certains groupes utilisent cette formule de manière très convaincante. L'autre extrême est la reconstitution complète, disons, d'un service liturgique, avec tout sauf le prêtre ${ }^{55}$

Qu'il s'agisse, au cœur de l'espace scénique, de recontextualiser la musique du Moyen Âge au moyen d'une mise en scène, ou de la transposer à la situation du concert, l'interprète réalise une démarche de sécularisation profane, au sens premier, en ce qu'il fait entrer dans le siècle présent la musique d'un autre temps.

Se pose également la question de la vraisemblance, notamment en ce qui concerne les timbres vocaux : un texte féminin doit-il obligatoirement être chanté par une voix de femme? La discographie répond négativement à cette question, puisqu'il arrive d'entendre des voix masculines interpréter de telles pièces... Mais résoudre cette question de la vraisemblance revient à répondre à celle, soulevée plus haut, des référents horsscène, lesquels construisent le regard et l'écoute du public. Réinvestir la subjectivité de l'œuvre médiévale telle que nous l'avons définie contribue à une "néo-médiévalité ${ }^{56}$ ». Adopter pour référent le personnage féminin, le trouvère, ou toute autre subjectivité, est un choix interprétatif, ce qui explique la diversité des propositions des ensembles spécialisés, chacun construisant son propre Moyen Âge, entre quête de vraisemblance historique et sensibilité artistique, dans une recherche de sens pour aujourd'hui.

91 Ainsi, le réinvestissement d'une subjectivité féminine à notre époque apparaît comme liée aux problématiques sociales actuelles. George Duby a souligné le rôle du présent dans l'intérêt porté, scientifiquement ou artistiquement, au Moyen Âge, notamment en ce qui concerne la place de la femme, en mutation dans notre société actuelle: «Dans la seule civilisation occidentale, et pour la première fois depuis les origines de l'espèce, la femme a cessé d'être considérée comme un être inférieur et nécessairement soumis à l'homme ${ }^{57}$ . » Dans ce contexte de mutation, interroger la féminité médiévale revient à l'éclairer de nos enjeux actuels, à la réinventer, voire la réhabiliter. Il se bâtit ainsi une poétique de la femme médiévale, rêvée, entrevue à travers un nouveau prisme, non plus celui des hommes, mais celui de la modernité. 


\begin{tabular}{|c|c|}
\hline Triplum & Triplum \\
\hline Je me dois bien doloseir & Je dois bien pleurer \\
\hline Et de chanchon faire reposeir & Et cesser de composer des chansons \\
\hline Cant celle ki mun cuer at pris at mari, & Car celle qui a pris mon cœur a un mari, \\
\hline Dont trop ai le cuer mari! & Ce qui attriste mon cœur! \\
\hline Nonporcant raison pieche at & C'est pour cette raison que pendant longtemps \\
\hline N'ous de chanteir. & Je n'ose chanter. \\
\hline Melhour bien m'en puis vanteir, & Je peux bien me vanter \\
\hline Car la belle mandeit m'at & De ce que la belle m’a révélé \\
\hline K'ultre sun greit & Qu'on lui a donné un mari \\
\hline Li at om mari doneit, & Contre son gré \\
\hline E ke ja ne l'amerat, & Et qu'elle ne l'aimera jamais \\
\hline Ne por ce ne laisserat & Ni ne renoncera \\
\hline Le bien ameir. & $\mathrm{Au}$ véritable amour. \\
\hline Anchois ferat & Elle fera en sorte \\
\hline Tant k'ameit bien se porat & Que celui qu'elle aimera \\
\hline Li siens clameir. & Puisse se dire bien aimé. \\
\hline Motetus & Motetus \\
\hline Por coi m'aveis vos doneit, & Pourquoi m'avez-vous donné \\
\hline Mere, mari? & Mère, un mari ? \\
\hline Cant ja par mun greit & Alors que jamais \\
\hline Ne fuist ensi & Je n'ai voulu \\
\hline K'a autrui fuisse doneie & Etre ainsi donnée \\
\hline K'a celi cui j'ai de moi saisit, & À un autre que celui que j'ai choisi, \\
\hline Ki tant m'a honoreie & Qui m’a si bien honorée \\
\hline C'onkes mais nus mieus ne deservit & Que nul n'a jamais mieux mérité \\
\hline K'amors li fuist graeie ; & Que je lui accorde mon amour; \\
\hline E vos l'en aveis a tort parti! & Et vous l'en avez à tort privé ! \\
\hline Diex ! J'astoie si bien assenneie, & Dieu ! J'étais si bien lotie, \\
\hline E vos m'aveis marïeie ! ainmi ! & Et vous m’avez mariée ! Hélas! \\
\hline Ja saviés vos bien k’avoie amis. & Vous saviez pourtant bien que j'avais un ami. \\
\hline Teneur & Teneur \\
\hline Docebit & Docebit \\
\hline
\end{tabular}


Mout me fu grief/Robin m'aime/Portare

\begin{tabular}{|c|c|}
\hline Texte original & Traduction \\
\hline Triplum & Triplum \\
\hline Mout me fu grief li departir & Ce me fut un grand chagrin que de quitter \\
\hline De m'amïete & Ma petite amie \\
\hline La jolie au cler vis, & La jolie au clair visage, \\
\hline Qui est blanche et vermellette & Qui est blanche et vermeille \\
\hline Comme rose par desus lis, & Comme une rose sur un lys, \\
\hline Ce m'est avis ; & Il me semble ; \\
\hline Son tres douz ris & Son très doux rire \\
\hline Mi fait fremir & Me fait tressaillir \\
\hline Et si oell vair riant languir. & Et son œil changeant et riant languir. \\
\hline Ha, Diex ! com mar la lessai! & Ha, Dieu ! Comme j'étais triste en la laissant! \\
\hline Blanchete comme flour de lis, & Jeune fille blanche comme une fleur de lys, \\
\hline Quant vous verrai? & Quand vous verrai-je? \\
\hline Dame de valour, & Dame de grande valeur, \\
\hline Vermelle comme rose en mai, & Vermeille comme la rose en mai, \\
\hline Pour vous sui en grant dolour. & J'éprouve à cause de vous une grande douleur. \\
\hline Motetus & Motetus \\
\hline Robin m'aime, Robin m'a & Robin m'aime, Robin m'a \\
\hline Robin m'a demandee & Robin m’a demandée : \\
\hline Si m'avra. & Ainsi, il m'aura. \\
\hline Robin m'achata corroie & Robin m'a acheté une ceinture \\
\hline Et aumonniere de soie ; & Et une aumônière de soie ; \\
\hline Pour quoi donc ne l'ameroie? & Pourquoi ne l'aimerais-je pas? \\
\hline Aleuriva! & Aleuriva! \\
\hline Robin m'aime, Robin m’a & Robin m'aime, Robin m’a \\
\hline Robin m'a demandee & Robin m’a demandée \\
\hline Si m’avra. & Ainsi, il m'aura. \\
\hline Teneur & Teneur \\
\hline Portare & Portare \\
\hline
\end{tabular}


S'on me regarde/prenes i garde/Hé ! Mi enfant

Texte original

Traduction 


\begin{tabular}{|c|c|}
\hline Triplum & Triplum \\
\hline S'on me regarde, & Si l'on me regarde, \\
\hline S'on me regarde, & Si l'on me regarde, \\
\hline Dites le moi ; & Dites-le moi ; \\
\hline Trop sui gaillarde, & Je suis trop gaillarde, \\
\hline Bien l'aperchoi. & Je le vois bien. \\
\hline Ne puis laissier que mon regard ne s'esparde, & Je ne peux empêcher que mon regard ne s'égare \\
\hline Car tes m'esgarde & Car un tel me regarde \\
\hline Dont mout me tarde & Dont il me tarde fort \\
\hline Qu'il m'ait o soi, & Qu'il m'ait auprès de lui, \\
\hline Qu'il a, en foi, & Car il a, en vérité, \\
\hline De m'amour plain otroi. & Le plein octroi de mon amour. \\
\hline Mais tel ci voi & Mais j'en vois ici un autre \\
\hline Qui est, je croi - & Qui est, je crois - \\
\hline Feu d'enfer l'arde ! - & Qu'il brûle en enfer !- \\
\hline Jalous de moi. & Jaloux de moi. \\
\hline Mais pour li d'amer ne recroi, & Mais je ne renoncerai pas à aimer pour lui, \\
\hline Car, par ma foi, & Car par ma foi, \\
\hline Pour nient m'esgarde ; & Il me regarde pour rien ; \\
\hline Bien pert sa garde : & Il perd bien sa garde : \\
\hline J'arai rechoi. & Je trouverai un refuge. \\
\hline Motetus & Motetus \\
\hline Prenés i garde & Faites attention \\
\hline S'on me regarde ; & Si l'on me regarde ; \\
\hline Trop sui gaillarde. & Je suis trop gaillarde. \\
\hline Dites le moi, & Dites-le moi, \\
\hline Pour Dieu, vous proi, & Pour Dieu, je vous en prie \\
\hline Car tes m'esgarde & Car un tel me regarde \\
\hline Dont mout me tarde & Dont il me tarde fort \\
\hline Qu'il m'ait o soi, & Qu'il m'ait auprès de lui, \\
\hline Bien l'aperchoi. & Je le vois bien. \\
\hline Et tel ci voi & Et j'en vois un \\
\hline Qui est, je croi & Qui est, je crois - \\
\hline Feu d'enfer l'arde ! - & Qu'il brûle en enfer ! - \\
\hline Jalous de moi. & Jaloux de moi. \\
\hline Mais pour li d'amer ne recroi. & Mais je ne renoncerai pas à aimer pour lui, \\
\hline Pour nient m'esgarde ; & Il me regarde pour rien ; \\
\hline TRien partitnsa gardpe : & Il perd bien sa garde : \\
\hline J'arai rechoi & Je trouverai un refuge \\
\hline Et de mon ami le dosnoi & Et j'aurai du plaisir avec mon ami \\
\hline Faire le doi : & Je dois le faire : \\
\hline
\end{tabular}


Je sui jonete et joliete/Hé Diex !/Veritatem

\begin{tabular}{|l|l}
\hline Texte original & Traduction
\end{tabular}




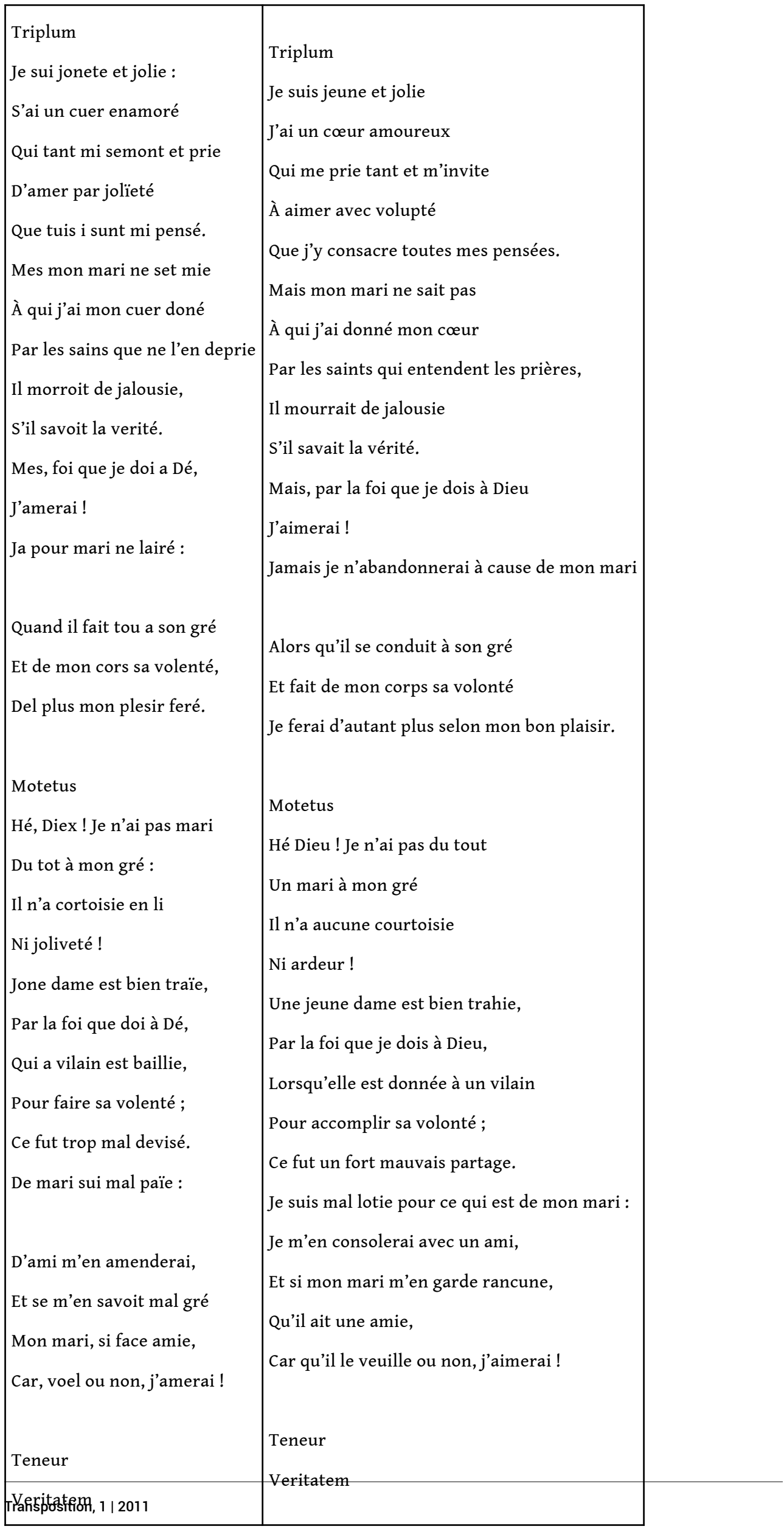




\section{BIBLIOGRAPHIE}

\section{Bibliographie}

\section{Articles}

BAL, Mieke, « Narration et focalisation. Pour une théorie des instances du récit », in Poétique, N 29, 1977, p. 107-127.

CASAGRANDE, Carla, « La femme gardée », in Histoire des femmes en Occident, t. 2, Le Moyen Âge, Paris, Plon, 1991, p. 83-116.

CULLIN, Olivier, « Motet », in FERRAND, Françoise (ed.), Guide de la musique du Moyen Âge, Paris, Fayard, 1999, p. 216.

KATAYAMA, Mikio, «La polyphonie textuelle dans le motet au XIII ${ }^{\mathrm{e}}$ siècle. Remarques sur quatre motets d'Adam de la Halle ", in Études de langue et littérature française, $\mathrm{N}^{\circ} 78$, Hakusuisha, Tokyo, 2001, p. 3-13.

MÖLK, Ulrich, « Chansons de femme, trobaïritz et la théorie romantique de la genèse et de la poésie lyrique romane ", in Lingua e stile, XXV, No 1, mars 1990, p. 135-146.

STANESCO, Michel, « Le texte primitif et la parole poétique médiévale », in BOUTET, Dominique, HARF-LANCNER, Laurence (ed.), Écriture et modes de pensée au Moyen Âge. VIII ${ }^{e}-X V^{e}$ siècles, Paris, Presses de l'École Normale Supérieure, 1993, p. 151-155.

\section{Ouvrages}

BARBA, Eugenio, Le canoë de papier, traité d'anthropologie théâtrale, Saussan, L'entretemps, 2004.

BAUMGARTNER, Emmanuèle, Le récit médiéval, Paris, Hachette, 1995.

BEC, Pierre, La lyrique française au Moyen Âge (XII ${ }^{e}$-XIII ${ }^{e}$ siècles). Contribution à une typologie des gens poétiques médiévaux, t. 1, Paris, Picard, 1977.

BENVENISTE, Émile, Problèmes de linguistique générale, vol. 1, Paris, Gallimard, 1966.

BERTHELOT, Anne, Histoire de la littérature française du Moyen Âge, Paris, Nathan, 1989.

CULLIN, Olivier, Laborintus : Essais sur la musique du Moyen Âge, Paris, Fayard, 2004.

DOSS-QUINBY, Eglal, TASKER GRIMBERT, Joan, PFEFFER, Wendy (et al.), Songs of the Women Trouvères, New Haven, London, Yale University Press, 2001.

DUBY, Georges, An 1000-An 2000. Sur les traces de nos peurs, Paris, Textuel, 1995.

EVERIST, Mark, French Motet in the Thirteenth Century: Music, Poetry and Genre, Cambridge, Cambridge University Press, 1994.

FERAL, Josette, Mise en scène et jeu de l'acteur : entretiens, t. 2 : le corps en scène, Montréal, Jeu.

FRITZ, Jean-Marie, Paysages sonores du Moyen Âge : le versant épistémologique, Paris, Honoré Champion, 2000. 
GENETTE, Gérard, Figures III, Paris, Seuil, 1972.

HOPPIN, Richard, La musique au Moyen Âge, Liège, Mardaga, 1991.

HUOT, Sylvia, Allegorical Play in the Old French Motet. The Sacred and the Profane in the ThirteenthCentury Polyphony, Stanford, Santford University Press, 1997.

JACOBSOHN, Ricardo E., Spectacle vivant, spectacle sur support. Esthétique comparée des arts du spectacle, Paris, L'Harmattan, 2006.

LE GOFF, Jacques, TRUONG, Nicolas, Une histoire du corps au Moyen Âge, Paris, Liana Levi, 2003.

MAUSS, Marcel, Sociologie et anthropologie, Paris, Presses Universitaires de France, 1968.

MONMERQUE, Louis Jean Nicolas, Théâtre français du Moyen Âge, publié d'après les manuscrits de la Bibliothèque du Roi, Paris, Firmin Didot, 1842.

PAGE, Christopher, The Owl and the Nightingale : musical life and ideas in France 1100-1300, Berkeley, University of California Press, 1992.

SAINT AUGUSTIN, AGAESSE, Paul (trad.), De trinitate, Paris, Desclée de Brouwer, 1955.

SCHMITT, Jean-Claude, Le corps, les rites, le rêve, le temps. Essais d'anthropologie médiévale, Paris, Gallimard, 2001.

SHERMAN, Bernard D., Inside early music. Conversation with performers, New York, Oxford University Press.

ZINK, Michel, La subjectivité littéraire autour du siècle de Saint Louis, Paris, Presses Universitaires de France.

ZUMTHOR, Paul, Introduction à la poésie orale, Paris, Seuil, 1983.

\section{Documents électroniques :}

CHANDES, Gérard, « Le Moyen Âge en jeu : conclusion », in Actes du colloque Le Moyen Âge en jeu, 2008 [en ligne]. Disponible sur : http://lapril.u-bordeaux3.fr/IMG/pdf/

Conclusion_Colloque_V3_pdf.pdf (consulté le 13/06/2010).

DE GROUCHY, Jean, De musica [en ligne]. Disponible sur : http://www.chmtl.indiana.edu/ tml/14th/GRODEM_TEXT.html (Consulté le 02/04/2010).

\section{ANNEXES}

\section{Textes et traduction ${ }^{58}$}

Je me dois bien doloseir/Por coi m'aveis vos doneit/Docebit

\section{NOTES}

1. BENVENISTE, Émile, Problèmes de linguistique générale, Paris, Gallimard, 1966, p. 269. 
2. GENETTE, Gérard, « Discours du récit », in Figures III. Paris, Seuil, coll. Poétique, 1972, p. 208.

3. BAL, Mieke, "Narration et focalisation. Pour une théorie des instances du récit », in Poétique, $\mathrm{N}^{\circ} 29,1977$, p. 107-127

4. MÖLK, Ulrich, « Chansons de femme, trobaïritz et la théorie romantique de la genèse et de la poésie lyrique romane », in Lingua e stile, XXV, N 1, mars 1990, p. 145.

5. CASAGRANDE, Carla, «La femme gardée ", in Histoire des femmes en Occident, t. 2, Le Moyen Âge, Paris, Plon, 1991, p. 113.

6. DOSS-QUINBY, Eglal, TASKER GRIMBERT, Joan, PFEFFER, Wendy (et al.), Songs of the Women Trouvères, New Haven, London, Yale University Press, 2001.

7. HOPPIN, Richard, La musique au Moyen Âge, Liège, Mardaga, 1991, p. 373.

8. Ibid., p. 378.

9. DE GROUCHY, Jean, De musica [en ligne]. Disponible sur: http:// www.chmtl.indiana.edu/tml/14... (Consulté le 02/04/2010). «Cantus autem iste non debet coram vulgaribus propinari, eo quod eius subtilitatem non animadvertunt nec in eius auditu delectantur, sed coram litteratis et illis, qui subtilitates artium sunt quaerentes. Et solet in eorum festis decantari ad eorum decorationem, quemadmodum cantilena, quae dicitur rotundellus, in festis vulgarium laicorum ».

10. PAGE, Christopher, The Owl and the Nightingale: musical life and ideas in France 1100-1300, Berkeley, University of California Press, 1992, p. 119: "performed when gens letrees that is to say learned clerics - were gathered together ».

11. DE NANGIS, Guillaume, cité in PAGE, Christopher, op. cit., p. 228 : «Les bourgois de Paris firent feste grant et solempnel, et encourtinerent la ville de riches dras de diverses couleurs et de pailes et de cendaulx. Lez dames et les pucelles sesbaudissoient en chantant diverses chançons et diverses motès. "

12. CULLIN, Olivier, "Motet ", in FERRAND, Françoise (ed.), Guide de la musique du Moyen Âge, Paris, Fayard, 1999, p. 216.

13. CULLIN, Olivier, Laborintus, essais sur la musique du Moyen Âge, Paris, Fayard, 2004.

14. LE GOFF, Jacques et TRUONG, Nicolas, Une histoire du corps au Moyen Âge, Paris, Liana Levi, 2003, p. 36.

15. SAINT AUGUSTIN, AGAESSE, Paul (trad.), De trinitate, XV, 11, § 20, Paris, Desclée de Brouwer, 1955, p. 471.

16. FRITZ, Jean-Marie, Paysages sonores du Moyen Âge: le versant épistémologique, Paris, Honoré Champion, 2000, p. 292.

17. SCHMITT, Jean-Claude, Le corps, les rites, le rêve, le temps. Essais d'anthropologie médiévale, Paris, Gallimard, 2001, p. 261.

18. MAUSS, Marcel, Sociologie et anthropologie, Paris, Presses Universitaires de France, 1968.

19. SCHMITT, Jean-Claude, op. cit., p. 257-258.

20. BEC, Pierre, La lyrique française au Moyen Âge (XII ${ }^{e}-X I I I^{e}$ siècles). Contribution à une typologie des genres poétiques médiévaux, t. 1, Paris, Picard, 1977, p. 17 et sq.

21. ZINK, Michel, La subjectivité littéraire autour du siècle de Saint Louis, Paris, Presses Universitaires de France, 1985.

22. HOPPIN, Richard, op. cit., p. 373. 
23. BEC, Pierre, op. cit.

24. Ibid., p. 62.

25. Ibid., p. 62-63.

26. Ibid., p. 70.

27. BENVENISTE, Émile, op. cit., chapitre XIX.

28. ZUMTHOR, Paul, Introduction à la poésie orale, Paris, Seuil, 1983, p. 135.

29. BEC, Pierre, op. cit., p. 19.

30. ZINK, Michel, op. cit., p. 50.

31. Cf. supra.

32. BEC, Pierre, op. cit., p. 17.

33. BERTHELOT, Anne, op. cit., p. 167.

34. BAUMGARTNER, Emmanuèle, Le récit médiéval, Paris, Hachette, 1995, p. 121.

35. HUOT, Sylvia, op. cit.

36. EVERIST, Mark, French Motet in the Thirteenth Century: Music, Poetry and Genre, Cambridge, Cambridge University Press, 1994.

37. FERAL, Josette, Mise en scène et jeu de l'acteur : entretiens, t. 2 : le corps en scène, 1999, p. 105.

38. BARBA, Eugenio, Le canoë de papier, traité d'anthropologie théâtrale, Saussan, L'entretemps, 2004, p. 41.

39. JACOBSOHN, Ricardo E., Spectacle vivant, spectacle sur support. Esthétique comparée des arts du spectacle, Paris, L'Harmattan, 2006, p. 65.

40. EVERIST, Mark, op. cit.

41. HUOT, Sylvia, op. cit.: "The motet, constructed of independent texts that are performed simultaneously, affords an opportunity for intertextual play that is unparalleled in other literary forms. »

42. Voir textes en annexe.

43. STANESCO, Michel, "Le texte primitif et la parole poétique médiévale », in BOUTET, Dominique, HARF-LANCNER, Laurence (ed.), Écriture et modes de pensée au Moyen Âge. VIII ${ }^{e}$ XV $V^{e}$ siècles, Paris, Presses de l'École Normale Supérieure, 1993, p. 153.

44. ZUMTHOR, Paul, op. cit., p. 149.

45. Ibid., p. 149.

46. KATAYAMA, Mikio, «La polyphonie textuelle dans le motet au XIII ${ }^{\mathrm{e}}$ siècle. Remarques sur quatre motets d'Adam de la Halle », in Études de langue et littérature française, $\mathrm{N}^{\circ} 78$, Hakusuisha, Tokyo, 2001, p. 12.

47. Ibid., p. 12.

48. MONMERQUE, Louis Jean Nicolas, Théâtre français du Moyen Âge, publié d'après les manuscrits de la Bibliothèque du Roi, Paris, Firmin Didot, 1842, p. 28.

49. KATAYAMA, Mikio, op. cit., p.3 et sqq.

50. Voir texte en annexe.

51. BENVENISTE, Émile, op. cit., p. 256.

52. Voir texte en annexe. 
53. BEC, Pierre, op. cit., p. 70.

54. STANESCO, Michel, op. cit., p. 134.

55. SHERMAN, Bernard D., Inside early music, conversation with performers, New York, Oxford University Press, 1997, p. 45. «There are several possible solutions. To illustrate one extreme, some ensemble present medieval music in the tradition of the nineteenthcentury song recital. They wear formal dress. They begin their concerts by bowing to the applause of their audiences. They sing a number of pieces, receiving applause after each work or "set". Their concert include an intermission, and may be followed by one or more encores. Some groups use this format very convincingly. The other extreme is the complete reconstruction of, say, a liturgical service with everything but the priest... »

56. CHANDES, Gérard, "Le Moyen Âge en jeu : conclusion ", in Actes du colloque Le Moyen Âge en jeu, 2008, [en ligne]. Disponible sur: http://lapril.u-bordeaux3.fr/IMG/pd... (consulté le 13/06/2010).

57. DUBY, Georges, An 1000-An 2000. Sur les traces de nos peurs, Paris, Textuel, 1995, p. 40.

58. Les traductions de ces textes ont été réalisées d'après les éditions d'Eglal DOSS-

QUINBY, Joan TASKER GRIMBERT, Wendy PFEFFER, (et al.), op. cit.

\section{RÉSUMÉS}

La spécificité du motet, genre polyphonique représentatif $d u$ XIII ${ }^{\mathrm{e}}$ siècle français, réside dans le principe de polytextualité sur lequel il se fonde : plusieurs textes y sont énoncés simultanément par les différentes voix. Le motet féminin intègre dans cette problématique la question du genre, en donnant la parole à des voix féminines seules, ou en interaction avec des voix masculines. Dans ce contexte, comment fonctionne la subjectivité du texte, et comment peut-elle être réinvestie par la voix de l'interprète?

En prolongement des travaux de Sylvia Huot et Mark Everist, nous interrogeons la polyphonie médiévale, sous la forme d'un corpus de motets féminins anonymes, non avec les outils de la musicologie, mais plutôt par à travers des notions de linguistique et d'anthropologie théâtrale, afin de définir un corps énonçant pour le motet féminin. La théorie d'Emile Benveniste, qui définit la subjectivité comme " la capacité du locuteur à se poser comme sujet ", apparaît comme un moyen de questionner les notions d'énonciation et de focalisation dans le cadre de ce type de discours fictif, à plusieurs voix. Cette analyse, qui revient à proposer une interprétation prenant en compte l'intertextualité, permet d'établir une typologie de la locution dans le motet féminin, propre à mettre en évidence une conception médiévale des notions de personnage, de subjectivité et de théâtralité dans le motet. Les définir amène à poser la question de l'interprétation du motet dans la société médiévale, qui l'a façonné, et dans la nôtre, qui réinvestit cette forme à travers la pratique du concert.

French polyphonic secular motets are based on polytextuality: several texts are simultaneously set out by several voices. Women's motets let female voices speak, possibly in interaction with male voices: polytextuality is consequently related to gender problems.

As an outcome to Sylvia Huot's and Mark Everist's works, we question a corpus of women's French motets through linguistic, anthropological and theatrical notions. How does the text's 
subjectivity work in such a context? How may it be invested in by performer's voices? Emile Benveniste's theory defines subjectivity as a "speaker's capacity to come up as a subject". Subjectivity seems a way of questioning notions of enunciation and focalization related to this type of fictional discourse. This analysis, which takes into account intertextuality, allows to establish a typology of women's locution in the motet which focuses the light on medieval conceptions of the character's subjectivity and theatricality. According to these conceptions, one may think about women's motets medieval and present-day performances.

\section{INDEX}

Mots-clés : énonciation, focalisation, interprète, motet, point de vue, subjectivité, Moyen Âge, trouvère, voix, chanson de femme, XIIIe siècle

Keywords : 13 th century, body, enunciation, focus, motet, performer, Middle Ages, point of view, subjectivity, trouvère, voice, woman's song

\section{AUTEUR}

\section{RACHEL MÉEGENS}

Doctorante à l'université d'Evry Val-d'Essonne, Rachel Méegens travaille depuis plusieurs années sur le thème de la musique médiévale et de son intégration aux pratiques culturelles actuelles, et prépare une thèse portant sur l'interprétation et la mise en spectacle des chansons des femmes trouvères. Egalement musicienne, elle interprète différents répertoires, et en particulier celui du Moyen Âge, en compagnie de plusieurs formations. 